Marcel Dekker Lecture Notes in Pure and

Applied Mathematics 231 (2002), pages 191-212

\title{
CONTROLLING THE ZERO DIVISORS OF A COMMUTATIVE RING
}

\author{
SARAH GLAZ \\ Department of Mathematics, University of Connecticut, Storrs, CT 06269 \\ glaz@uconnvm.uconn.edu
}

\begin{abstract}
This article explores nine zero divisor controlling conditions, their impact on the domain-like behavior of rings with zero divisors, their interrelations, and their applications to the extension of domain properties to rings with zero divisors.
\end{abstract}

\section{INTRODUCTION}

Commutative algebraists often consider the following question: given a property $\mathrm{P}$ that holds for a domain, how best can one extend $\mathrm{P}$ to rings with zero divisors? The answer may sometimes depend completely on the particular property. But more often, especially when $\mathrm{P}$ involves all the entities of a ring $\mathrm{R}$, where by entities we mean elements or ideals or finitely generated ideals, etc., one may take one of the following three approaches:

a. Require that all entities of R satisfy $\mathrm{P}$, and place no other restrictions on R.

b. Require that all regular entities of R satisfy P.

c. Require that all entities of R satisfy $\mathrm{P}$, and place some conditions on R to control the behavior of its zero divisors, that is, to make $\mathrm{R}$ share certain characteristics of a domain.

Each of these approaches have been used for various properties $\mathrm{P}$ with certain degrees of success. Which approach one uses depends on personal taste, and on the desired properties of the generalization. For example, if one wishes to 
have some control and knowledge of the behavior of non regular entities of the ring, one will try to avoid approach (b), which puts no restrictions on the non regular entities of the ring. Approach (a) is not always feasible, as certain conditions cannot be imposed on non regular entities without additional hypotheses.

The aim of this article is to consider a large number of zero divisor controlling conditions found in the literature. Some of these conditions were used in generalizing a domain property to rings with zero divisors using approach (c); other of these conditions have interesting and deep implications on the nature of the ring itself and were investigated in their own right. In each case, we will explore briefly the origin of the condition and its use for generalizations of domain properties to a ring $\mathrm{R}$ with zero divisors. We will point out the impact of each condition on sets of zero divisors, localizations by prime ideals, total ring of quotients, or sets of minimal prime ideals of the ring, all of which determine the closeness of the ring behavior to that of a domain. To the extent that it is possible, we will point out the relations between the various conditions, and provide examples and counterexamples.

Throughout the paper, all rings are commutative with identity. We will use the following notation and basic notions regarding a ring $\mathrm{R}$ :

\section{divisors of $R$.}

$$
\mathrm{Z}(\mathrm{R})=\{\mathrm{a} \in \mathrm{R} \mid \mathrm{ax}=0 \text { for some } 0 \neq \mathrm{x} \in \mathrm{R}\} \text { denotes the set of zero }
$$

\section{nilpotent elements of $R$.}

$\operatorname{Nil}(R)=\left\{a \in R \mid a^{n}=0\right.$ for some positive integer $\left.n\right\}$ denotes the set of

$$
\operatorname{Min}(R)=\{P \in \operatorname{spec} R \mid P \text { is a minimal prime ideal of } R\} \text { denotes the set }
$$

\section{of minimal primes of $R$.}

$\mathrm{Q}(\mathrm{R})$ denotes the total ring of quotients of $\mathbf{R}$, that is, the localization of $\mathrm{R}$ by the set of all its non zero divisors.

A non zero divisor of $\mathrm{R}$ will be called a regular element, and an ideal of $\mathrm{R}$ which contains a regular element will be called a regular ideal.

The oldest and most extensively studied zero divisor controlling condition is that which asks $\mathrm{R}$ to be a reduced ring, that is, a ring with no nonzero nilpotent elements. The literature pertaining to the nature of reduced rings is too vast to mention. For that reason, we will consider this condition only when it appears in the context of the other zero divisor controlling conditions considered in this paper. We will explore the following zero divisor controlling conditions on a ring $\mathrm{R}$. Several other, less used conditions will show up throughout the exposition.

\section{1. $\mathrm{R}$ is locally a domain.}


2. $Q(R)$ is a Von Neumann regular ring.

3. $\operatorname{Min}(\mathrm{R})$ is a compact subspace of $\operatorname{Spec}(\mathrm{R})$ in the Zariski topology.

4. $\mathrm{R}$ is a PP ring (sometimes called a weak Baer ring).

5 . $\mathrm{R}$ is a ring with few zero divisors.

6 . $\mathrm{R}$ is an additively regular ring.

7. $\mathrm{R}$ is a Marot ring.

8. $R$ is a $Z D$ ring.

9. Every zero divisor of $\mathrm{R}$ is nilpotent.

The interrelations between these nine properties makes it hard to separate the exposition into independent sections. Nevertheless, for the sake of readability, we divided the conditions into two groups. Section 2 considers conditions 1 - 4, and Section 3 considers conditions 5 - 9. Thus, Sections 2 and 3 explore the conditions themselves and the relations between them. Section 4 is devoted to a representative sample of applications of the zero divisor controlling conditions to the extension of domain properties to rings with zero divisors. We cover briefly the extended notions of valuation, Prufer and Krull rings; finite conductor, quasi- coherent and G-GCD rings; and Going Down and related rings. Interested readers are provided with a bibliography for further reading on each topic.

\section{MIN(R) AND LOCALIZATIONS OF R} a domain.

We first consider conditions under which a commutative ring $\mathrm{R}$ is locally

THEOREM 2.1 [G1], [M1], [M2] Let $R$ be a ring. The following conditions are equivalent:

1. $R_{P}$ is a domain for every prime ideal $P$ of $R$.

2. $R_{m}$ is a domain for every maximal ideal $m$ of $R$.

3. Every principal ideal of $R$ is flat.

4. $R$ is reduced and every prime ideal of $R$ contains a unique minimal prime ideal.

5. $R$ is reduced and every maximal ideal $m$ of $R$ contains a unique minimal prime ideal $P$.

In this case, $P=\{r \in R \mid$ there is a $u \in R-m$ such that $u r=0\}$ and

$R_{P}=Q\left(R_{m}\right)$, the quotient field of $R_{m}$.

This theorem appears with somewhat different proofs in Matlis [M2], and in Glaz [G1]. (4) does not appear in either of these sources, but appears in 
[M1], along with a few other of the conditions of this theorem, proved under the restriction that $\mathrm{Q}(\mathrm{R})$ is Von Neumann regular. As this assumption is unnecessary, we included (4) here. Rings satisfying that every principal ideal is flat are sometimes called PF (or PIF) rings.

EXAMPLE 2.2 The following examples are taken from several sources: [E], [G1], [M1], [M2], [Q1], [Q2], and [V]:

1. Coherent local rings satisfying that every principal ideal has finite projective dimension are locally domains.

2. Coherent regular rings, that is, coherent rings satisfying that every finitely generated ideal has finite projective dimension, are locally domains. In particular, this class of rings includes all coherent rings $\mathrm{R}$ of finite weak global dimension. It is worthwhile mentioning that the class of coherent rings of finite weak global dimension includes the classical non Noetherian rings such as Von Neumann regular, semihereditary, and hereditary rings. Von Neumann regular rings are rings $R$ satisfying that for every $a \in R$, there is $a b \in R$ such that $a b^{2}=a$. Equivalently, $\mathrm{R}$ is Von Neumann regular iff w.gl.dim $\mathrm{R}=0$. Such rings are automatically coherent. Semihereditary rings are rings in which every finitely generated ideal is projective. Equivalently, $\mathrm{R}$ is semihereditary iff $\mathrm{R}$ is coherent and w.gl.dim $\mathrm{R} \leq 1$. Hereditary rings are rings with gl.dim $\mathrm{R}=1$. They are always coherent.

3. Rings of global dimension 2 are locally domains.

4. A ring $R$ has w.gl.dim $R \leq 1$ iff $R_{P}$ is a valuation domain for every prime ideal $\mathrm{P}$ of $\mathrm{R}$. Therefore rings $\mathrm{R}$ with w.gl.dim $\mathrm{R} \leq 1$ are always locally domains, regardless of their coherence status.

We next consider the condition: $\operatorname{Min}(\mathrm{R})$ is compact in the induced Zariski topology from $\operatorname{Spec}(R)$. If $R$ is Noetherian or a domain $\operatorname{Min}(R)$ is finite and therefore compact. In general $\operatorname{Min}(\mathrm{R})$ does not have to inherit the compactness of $\operatorname{Spec}(\mathrm{R})$. For a reduced ring one has a way of testing the minimality of a prime ideal $P$.

PROPOSITION 2.3 Let $R$ be a reduced ring, and let $P$ be a prime ideal of $R$. Then $P$ is a minimal prime ideal iff for all $x \in P,(0: x) \not \subset P$.

As a consequence of this proposition for a reduced ring $\mathrm{R}, \operatorname{Min}(\mathrm{R})$ is a Hausdorff space in the induced Zariski topology, but it still might not inherit the compactness of $\operatorname{Spec}(\mathrm{R})$. 
We exhibit a number of conditions under which $\operatorname{Min}(\mathrm{R})$ is indeed compact when $\mathrm{R}$ is a reduced ring.

THEOREM 2.4 [G1], [M2] Let $R$ be a reduced ring, and let $S$ be a ring extension of $R$. Then:

1. If every prime ideal of $S$ contracts to a minimal prime ideal of $R$, then $\operatorname{Min}(R)$ is compact.

2. If $S$ is Von Neumann regular and flat over $R$, then every prime ideal of $S$ contracts to a minimal prime ideal of $R$. Hence, in this case, $\operatorname{Min}(R)$ is compact.

COROLLARY 2.5 [G1], [M2], [Q1] Let $R$ be a reduced ring with $Q(R)$, the total ring of quotients of $R$, Von Neumann regular. Then $\operatorname{Min}(R)$ is compact.

EXAMPLE 2.6 Quentel [Q1] provides an example of a reduced ring with compact minimal spectrum, but not Von Neumann regular total ring of quotients. The version presented below is from [G1]:

Let $K$ be a countable algebraically closed field, and let $I$ be an infinite set. Denote by $K^{I}$ the set of all set maps from $I$ to $K$. For a map $f \in K^{I}$, let supp $f=\{a \in I \mid f(a) \neq 0\}$, and let cosupp $f=\{a \in I \mid f(a)=0\}$. A $K$ subalgebra $R$ of $K^{I}$ is called a $T$ algebra if it satisfies the following two conditions:

(i) $R$ is countable and contains all the constant maps.

(ii) Every $f \in R$ which is not constant satisfies cosupp $f \neq \phi$.

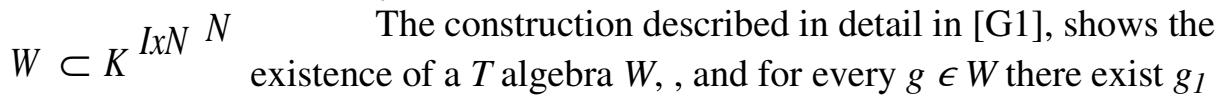
and $g_{2} \epsilon W$ such that cosupp $g=\operatorname{supp} g_{1} \cup \operatorname{supp} g_{2}$. Such an algebra $W$ is a reduced ring equal to its own total ring of quotients, it has compact $\operatorname{Min}(W)$, but it is not Von Neumann regular.

Before presenting the exact connection between the compactness of $\operatorname{Min}(\mathrm{R})$, the Von Neumann regularity of $\mathrm{Q}(\mathrm{R})$, and the locally domain property of $R$, we will present several other conditions under which $\operatorname{Min}(R)$ is compact or $\mathrm{Q}(\mathrm{R})$ is Von Neumann regular when $\mathrm{R}$ is a reduced ring.

THEOREM 2.7 [G1], [M2], [O], [Q1] Let $R$ be a reduced ring. The following conditions are equivalent:

1. $\operatorname{Min}(R)$ is compact.

2. For every element $b \in R$, there exists a finitely generated ideal $J \subset(0: b)$ such that $(0: b R+J)=0$. 
3. $\Pi R_{P}$, where $P$ runs over Min $(R)$, is a flat $R$ module.

4. $E(R)$, the injective envelope of $R$, is a flat $R$ module.

5. $M(R)$, the maximal flat epimorphic extension of $R$, is Von Neumann regular.

Theorem 2.7 collects a number of conditions, scattered throughout the mentioned sources, under which $\operatorname{Min}(\mathrm{R})$ is compact for a reduced ring $\mathrm{R}$. To clarify the statements of this theorem, we remind the reader of the definitions of $\mathrm{E}(\mathrm{R})$ and $\mathrm{M}(\mathrm{R})$ mentioned in (4) and (5).

Let $\mathrm{R}$ be a ring and let $\mathrm{M}$ be an $\mathrm{R}$ module. An $\mathrm{R}$ module $\mathrm{E}$ is called an essential extension of $M$, if $M \subset E$, and for any nonzero submodule $E^{\prime}$ of $E$ we have $\mathrm{E}^{\prime} \cap \mathrm{M} \neq 0$. Every $R$ module admits an essential injective extension $\mathrm{E}(\mathrm{M})$, which is unique up to isomorphism. This extension is called the injective envelope of $M$. If $E(M)$ is the injective envelope of $M$, there is no injective proper submodule between $\mathrm{M}$ and $\mathrm{E}(\mathrm{M})$. Matlis [M2] approaches both the compactness of $\operatorname{Min}(R)$ and the Von Neumann regularity of $Q(R)$ via the exploration of $\mathrm{E}(\mathrm{R})$.

Let $\mathrm{R}$ be a ring. Denote by $\mathrm{M}(\mathrm{R})$, the maximal flat epimorphic extension of $\mathbf{R}$, the unique (up to isomorphism) ring satisfying:

1. $R \subset M(R)$, and $M(R)$ is a flat epimorphism of $R$.

2. If $R \subset S$ and $S$ is a flat epimorphism of $R$, then $S \subset M(R)$.

The approach to the compactness of $\operatorname{Min}(\mathrm{R})$ and to the Von Neumann regularity of $\mathrm{Q}(\mathrm{R})$ via the investigation of $\mathrm{M}(\mathrm{R})$ is due to Quentel [Q1] and Olivier [O], [O1].

EXAMPLES 2.8 The above theorem guarantees that if $\mathrm{R}$ is a reduced coherent ring, then $\operatorname{Min}(\mathrm{R})$ is compact.

The next theorem collects the conditions under which the total ring of quotients of a reduced ring is Von Neumann regular.

THEOREM 2.9 [G1], [H], [M2], [O], [Q1] Let $R$ be a reduced ring. The following conditions are equivalent:

1. $Q(R)$ is a Von Neumann regular ring.

2. If $I$ is an ideal of $R$ contained in the union of the minimal prime ideals of $R$, then I is contained in one of them.

3. If $J$ is a finitely generated ideal of $R$, then there exists $a b \in J$ and an $a \in(0: J)$ such that $a+b$ is a regular element of $R$.

4. If $b \in R$, then there exists $a \in(0: b)$ such that $(0: a R+b R)=0$.

5. $Q(R)=M(R)$. 
6. Min $(R)$ is compact and if a finitely generated ideal is contained in the union of the minimal prime ideals of $R$, then it is contained in one of them.

7. $\operatorname{Min}(R)$ is compact and each finitely generated ideal consisting entirely of zero divisors has a nonzero annihilator.

EXAMPLE 2.10 The above theorem guarantees that if $\mathrm{R}$ is a coherent regular ring, then $\mathrm{Q}(\mathrm{R})$ is Von Neumann regular.

At this stage we are ready to present the main result connecting the zero divisor controlling conditions presented in this section.

A ring $\mathrm{R}$ is called a PP ring (or a weak Baer ring) if every principal ideal of $\mathrm{R}$ is projective.

THEOREM 2.11 [G1] Let $R$ be a ring. The following conditions are equivalent: 1. $\operatorname{Min}(R)$ is compact and every principal ideal of $R$ is flat.

2. $R$ is a PP ring.

3. $Q(R)$ is Von Neumann regular and every principal ideal of $R$ is flat.

The ideas for the proof of this theorem also appear in [Q1] and [V], excellent sources, where some errors crept into the approach to this particular result. A correct version appears in [G1].

EXAMPLES 2.12 Two examples [G1] show that the two conditions in (1), and the two conditions in (3) of Theorem 2.11, are independent of each other.

1. [V] Let $\mathrm{M}$ be a countable direct sum of copies of $\mathrm{Z} / 2 \mathrm{Z}$ with addition and multiplication defined componentwise. Let $\mathrm{R}=\mathrm{Z} \oplus \mathrm{M}$ and define multiplication as follows: for $\mathrm{m}, \mathrm{n} \in \mathrm{M}$ and $\mathrm{a}, \mathrm{b} \in \mathrm{Z}$ $(\mathrm{a}, \mathrm{m})(\mathrm{b}, \mathrm{n})=(\mathrm{ab}, \mathrm{an}+\mathrm{bm}+\mathrm{mn})$. Every principal ideal of $\mathrm{R}$ is flat, but $\operatorname{Min}(\mathrm{R})$ is not compact.

2. [M2] Let $\mathrm{R}$ be a Noetherian, local, reduced ring which is not a domain. Then $\operatorname{Min}(\mathrm{R})$ is compact (actually finite), and $\mathrm{Q}(\mathrm{R})$ is a finite direct sum of the domains

$R_{P}$, where $P$ runs over Min $(R)$. Therefore $Q(R)$ is Von Neumann regular, but $R$ is not a domain, so not every principal ideal of $\mathrm{R}$ is flat.

PP rings appear in the literature in expected and unexpected places. From Theorem 2.11, we know that such rings are locally domains and possess Von Neumann regular total ring of quotients. Another way of looking at such a ring is 
observing that a principal ideal aR is projective iff $(0: \mathrm{a})$ is generated by an idempotent. Hence PP rings have plenty of idempotents. In fact, every element $\mathrm{a} \in \mathrm{R}$ can be expressed as $\mathrm{a}=\mathrm{a}^{\prime} \mathrm{e}$, where $\mathrm{a}^{\prime}$ is a non zero divisor in $\mathrm{R}$, and $\mathrm{e}$ is an idempotent in R. Another useful property of PP rings is:

THEOREM 2.13 [G3] Let $R$ be a PP ring, and let I be a finitely generated flat ideal of $R$. Then I is projective.

There are many PP rings, for example, all coherent regular rings are such, and in the application section, we will exhibit a class of not necessarily coherent PP rings, namely G-GCD rings.

\section{MAROT RINGS AND RELATED CONDITIONS}

The notion of a ring with few zero divisors was introduced by Davis [D]. A maximal ideal of zero is an ideal (necessarily prime) maximal with respect to not containing regular elements. The set of zero divisors of $\mathrm{R}, \mathrm{Z}(\mathrm{R})=\cup \mathrm{P}$, as $\mathrm{P}$ runs over all maximal ideals of zero of $R$. A ring $R$ is said to have few zero

divisors if it has only finitely many maximal ideals of zero, equivalently, $Z(R)$ is a union of finitely many prime ideals.

Because of the one-to-one correspondence between the prime ideals of a ring $R$ which contain no regular elements and the prime ideals of $Q(R)$, it is evident that $R$ has few zero divisors iff $Q(R)$ is semilocal. It follows that if $R$ has few zero divisors, then any overring of $\mathbf{R}$, that is, any ring between $R$ and $Q(R)$, has few zero divisors. In particular, any overring of a Noetherian ring has few zero divisors, providing a large family of examples of rings of this kind.

A ring $\mathrm{R}$ with total ring of quotients $\mathrm{Q}(\mathrm{R})$ is said to be additively regular if for each $\mathrm{z} \in \mathrm{Q}(\mathrm{R})$, there exists $a \mathrm{u} \in \mathrm{R}$ such that $\mathrm{z}+\mathrm{u}$ is a regular element in $\mathrm{Q}(\mathrm{R})$. This condition appears first in [M1], and is named in [GH].

PROPOSITION $3.1[\mathrm{GH}]$ Let $R$ be a ring. The following conditions are equivalent:

1. $R$ is additively regular.

2. For each $z \in Q(R)$ and each regular element $b$ of $R$, there exists a $u \in R$ such that $z+$ bu is a regular element of $Q(R)$.

3. For each $a \in R$ and each regular element $b$ of $R$, there exists $a u \in R$ such that $a+u b$ is regular in $R$. 
Additively regular rings have the following useful property:

THEOREM 3.2 [M3] Let $R$ be an additively regular ring. Let $I_{1}, \ldots, I_{n}$ and $I$ be regular ideals of $R$. Denote by Reg(I) the set of regular elements of I. Then $\operatorname{Reg}(I) \subset \cup\left\{I_{i} \mid 1 \leq i \leq n\right\}$ iff $I \subset \cup\left\{I_{i} \mid 1 \leq i \leq n\right\}$.

THEOREM 3.3 [H1], [M1] Let $R$ be a ring with total ring of quotients $Q(R)$. If $Q(R) / N i l(Q(R))$ is Von Neumann regular, then $R$ is additively regular. In particular, if $Q(R)$ is Von Neumann regular, then $R$ is additively regular.

\section{EXAMPLES 3.4}

1. [PS] It follows from Theorem 3.3 that any ring whose total ring of quotients has Krull dimension zero is additively regular.

2. [M1] For any ring $R$, the polynomial ring in any number of variables is an additively regular ring.

3. $[\mathrm{GH}]$ Let $\mathrm{R}=\prod \mathrm{R}_{\alpha}$ for an arbitrary set $\{\alpha\}$. Then $\mathrm{R}$ is an additively regular ring iff $R_{\alpha}$ is an additively regular ring for every $\alpha$.

A ring $\mathrm{R}$ is called a Marot ring if every regular ideal can be generated by a set of regular elements. This property was defined by Marot [M1].

THEOREM 3.5 [M1] Let $R$ be a ring with total ring of quotients $Q(R)$. The following conditions are equivalent:

1. $R$ is a Marot ring.

2. Any two-generated ideal $(a, b)$ with $b$ regular can be generated by a finite set of regular elements.

3. Every regular fractional ideal of $R$, that is, every regular $R$ module contained in $Q(R)$, can be generated by a set of regular elements.

The next result connects the zero divisor controlling conditions exhibited, so far, in this section.

THEOREM 3.6 [D], [H], [M1] Let $R$ be a ring. Consider the following conditions:

1. $R$ has few zero divisors.

2. $R$ is an additively regular ring.

3. $R$ is a Marot ring. 
Then $(1) \Rightarrow(2) \Rightarrow(3)$.

None of the implications of Theorem 3.6 is reversible, as the two examples below show.

EXAMPLE $3.7[\mathrm{H}]$ There are additively regular rings which do not have few zero divisors.

Let $\left\{\mathrm{R}_{\alpha}\right\}$ be an infinite family of rings with few zero divisors. The product ring $\mathrm{R}=\Pi \mathrm{R}_{\alpha}$ is an additively regular ring which is not a ring with few zero divisors.

EXAMPLE 3.8 [H], [M3] There are Marot rings which are not additively regular.

The following example was constructed by Matsuda [M3]. Let $\mathrm{k}$ be a finite field of characteristic $\mathrm{p}>0$. Let $\mathrm{A}$ be the subring of the polynomial ring $\mathrm{k}[\mathrm{x}], \mathrm{A}=\mathrm{k}\left[\mathrm{x}^{\mathrm{p}}, \mathrm{x}^{\mathrm{p}+1}, \mathrm{x}^{\mathrm{p}+2}, \ldots\right]$. Let $\left\{\mathrm{F}_{0}, \mathrm{~F}_{1}, \ldots, \mathrm{F}_{\mathrm{n}}, \mathrm{G}_{1}, \mathrm{G}_{2}, \ldots\right\}$ be a set of irreducible polynomials in $\mathrm{k}[\mathrm{x}]$ such that:

1. $\mathrm{F}_{0}=\mathrm{x}$, and $\mathrm{F}_{1}=1+\mathrm{x}$.

2. $\operatorname{deg} \mathrm{F}_{\mathrm{i}}<2 \mathrm{p}$ for all $\mathrm{i}$.

3. $\operatorname{deg} \mathrm{G}_{\mathrm{j}} \geq 2 \mathrm{p}$ for all $\mathrm{j}$.

4. No two elements of the set are associated.

5. Each irreducible element of $\mathrm{k}[\mathrm{x}]$ is associated with an element of the set.

Let $\mathrm{K}_{\mathrm{j}}=\mathrm{k}[\mathrm{x}] /\left(\mathrm{G}_{\mathrm{j}}\right)$. Then $\mathrm{K}_{\mathrm{j}}$ is naturally an A module. Let $\mathrm{M}$ be the direct sum of the modules $K_{j}$, and let $R=A \alpha M$ be the trivial ring extension of $A$ by $\mathbf{M}$ (sometimes called the idealization of $\mathbf{M}$ in $\mathbf{A}[\mathrm{H}]$ ), that is, $\mathrm{R}$ is the set $\mathbf{A} \oplus \mathbf{M}$ with addition defined componentwise and multiplication defined by $(a, m)\left(a^{\prime}, m^{\prime}\right)=\left(a a^{\prime}, a m^{\prime}+a^{\prime} m\right)$ for all $a, a^{\prime} \in A$ and $m, m^{\prime} \in M$. Then $R$ is a Marot ring for any $\mathrm{p}$. For $\mathrm{p}=2$, for example, $\mathrm{R}$ is not an additively regular ring.

It is interesting to note that Matsuda's exploration of the additively regular and Marot properties of trivial ring extensions also yielded an example of a ring satisfying the condition of Theorem 3.2, but which is not additively regular.

EXAMPLE 3.9 [H], [M1], [SP] In addition to Noetherian rings, domains, and all additively regular rings, Marot rings can be generated in several other ways: 
1. Any overring of a Marot ring is a Marot ring.

2. $\mathrm{R}=\mathrm{R}_{1} \oplus \ldots \oplus \mathrm{R}_{\mathrm{n}}$ is a Marot ring iff $\mathrm{R}_{\mathrm{i}}$ is a Marot ring for every $\mathrm{i}$.

3. If every regular finitely generated ideal of a ring $\mathrm{R}$ is principal, then $\mathrm{R}$ is a Marot ring.

We now present a few other properties of Marot rings which makes this condition particularly useful when generalizing domain properties to rings with zero divisors.

An ideal $\mathrm{P}$ of a ring $\mathrm{R}$ is prime (respectively, primary) for its regular elements if whenever $a$ and $b$ are regular elements of $R$ such that $a b \in P$, then $\mathrm{a} \in \mathrm{P}$ or $\mathrm{b} \in \mathrm{P}$ (respectively, then $\mathrm{a} \in \mathrm{P}$ or $\mathrm{b}^{\mathrm{n}} \in \mathrm{P}$ for some positive integer $\mathrm{n}$ ).

THEOREM $3.10[\mathrm{H}],[\mathrm{SP}]$ Let $R$ be a Marot ring. Then a regular ideal $P$ of $R$ is prime (respectively, primary) iff $P$ is prime (respectively, primary) for its regular elements.

If a ring contains zero divisors, one may define invertibility of nonzero (fractional) ideals I of $\mathrm{R}$, that is of $\mathrm{R}$ submodules of $\mathrm{Q}(\mathrm{R})$, in a way that resembles the definition for the case $\mathrm{R}$ is a domain. Let $\mathrm{I}^{-1}=\{\mathrm{x} \in \mathrm{Q}(\mathrm{R}) \mid \mathrm{xI} \subset \mathrm{R}\}$ denote the inverse of $\mathrm{I}$. Then $\mathrm{I}$ is invertible if $\mathrm{II}^{-1}=\mathrm{R}$. The relation between invertibility, projectivity, and the property of being locally principal of an ideal can be summarized as follows:

PROPOSITION 3.11 Let $R$ be a ring and let I be an ideal of $R$. Then:

1. If I is invertible, then I is projective.

2. If I is projective, then I is locally principal.

3. If I is a finitely generated regular ideal, then I is invertible iff I is projective, iff I is locally principal. (In particular, if $R$ is a domain, 3 holds for every nonzero finitely generated ideal I of $R$.)

A (fractional) ideal I of $\mathrm{R}$ is divisorial if $\left(\mathrm{I}^{-1}\right)^{-1}=\mathrm{I}$.

Let $\mathrm{I}$ be a regular (fractional) ideal of $\mathrm{R}$ satisfying that $\mathrm{dI} \subset \mathrm{R}$ for some regular element $d \in R$. Then $I \subseteq\left(I^{-1}\right)^{-1} \subseteq \cap\{R a \mid I \subseteq R a\}$. Note that since $I$ is regular, the ideals $\mathrm{Ra}$ are necessarily regular. Hence, if $\mathrm{I}=\cap\{\mathrm{Ra} / \mathrm{I} \subseteq \mathrm{Ra}\}$, then $\mathrm{I}$ is divisorial. If $\mathrm{I}$ is generated by regular elements, say $\mathrm{I}=\Sigma \mathrm{Ra}_{\alpha}$, then $\mathrm{I}^{-1}=\cap \mathrm{Ra}_{\alpha}{ }^{-1}$. It follows that: 
PROPOSITION 3.12 [AM1] Let $R$ be a ring. Then $\left(I^{-1}\right)^{-1}=\cap\{R a \mid I \subseteq R a\}$ for every regular (fractional) ideal I of $R$ satisfying $d I \subset R$ for some regular element $d \epsilon R$ iff for every regular (fractional) ideal I of $R$ satisfying $d I \subset R$ for some regular element $d \epsilon R$, there exists a (fractional) ideal $J$ generated by regular elements and satisfying $d J \subset R$ for some regular element $d \epsilon R$, such that $\left(I^{-1}\right)^{-1}=\left(J^{-1}\right)^{-1}$.

COROLLARY 3.13 [AM1] Let $R$ be a Marot ring, and let I be a regular (fractional) ideal of $R$ satisfying $d I \subset R$ for some regular element $d \epsilon R$. Then $I$ satisfies $\left(I^{-1}\right)^{-1}=\cap\{R a \mid I \subseteq R a\}$.

D. D. Anderson and Markanda provide an example in [AM1] that shows that if $\mathrm{R}$ is not a Marot ring, then the conclusion of Corollary 3.13 does not need to hold.

A related zero divisor controlling condition was defined by Evans [E1]. A ring $R$ is called $\mathbf{a} Z \mathbf{Z D}$ ring, if $R / I$ is a ring with few zero divisors for every ideal I of R. Examples of $\mathrm{ZD}$ rings abound. Below is a sample found in the literature.

\section{EXAMPLES 3.14}

1.Noetherian rings are $\mathrm{ZD}$ rings.

2. [E1] A ring is called Laskerian if every ideal is the intersection of a finite number of primary ideals. Laskerian rings are $\mathrm{ZD}$ rings.

3. [E1] Localizations of ZD rings are $\mathrm{ZD}$ rings.

4. $[\mathrm{HO}] \mathrm{A}$ ring $\mathrm{R}$ is Noetherian iff the polynomial ring $\mathrm{R}[\mathrm{x}]$ is a $\mathrm{ZD}$ ring.

It is interesting to note that if the power series ring $\mathrm{R}[[\mathrm{x}]]$ is a $\mathrm{ZD}$ ring, $\mathrm{R}$ may not be Noetherian, but has Noetherian prime spectrum $[\mathrm{GH}]$.

We conclude this section with a different zero divisor controlling condition defined by Dobbs [D1]. Let $\mathrm{R}$ be a ring. In general, $\mathrm{Nil}(\mathrm{R}) \subseteq \mathrm{Z}(\mathrm{R})$. On one end of the spectrum $\operatorname{Nil}(\mathrm{R})=0$, that is, $\mathrm{R}$ is reduced. The other extreme is to ask that every zero divisor of $\mathrm{R}$ be nilpotent, that is $\mathrm{Nil}(\mathrm{R})=\mathrm{Z}(\mathrm{R})$. This condition is equivalent to 0 being a primary ideal of $R$.

Let $R$ be a ring in which 0 is a primary ideal. Then $R$ has a unique minimal prime ideal $P$, and $R_{P}=Q(R)$, the total ring of quotients of $R$. On the 
other hand, if $\mathrm{R}$ is a ring with a unique prime ideal, then 0 is a primary ideal of $\mathrm{R}$. This observation [D1] lead to a number of examples of rings in which 0 is a primary ideal.

EXAMPLES 3.15 [D1] Examples of rings R satisfying $\mathrm{Nil}(\mathrm{R})=\mathrm{Z}(\mathrm{R})$ :

1. Artinian local rings.

2. Let $\mathrm{A}$ be a domain, and let a be a nonzero prime element of $\mathrm{A}$. Then the ring $\mathrm{R}=\mathrm{A} /\left(\mathrm{a}^{2}\right)$ has 0 primary ideal.

\section{APPLICATIONS}

In this section, we will exhibit applications of the zero divisor controlling conditions discussed in Sections 2 and 3 to extension of domain properties to rings with zero divisors. The literature in this direction is vast. The main scope of this article is the exploration of the zero divisor controlling conditions themselves. We therefore restrict ourselves to a representative sample of applications which we describe without going into too many details.

\subsection{Valuation Rings, Prufer Rings, and Krull Rings}

Valuation rings with zero divisors were defined by Manis [M]. A valuation is a map $v$ from a ring $\mathrm{K}$ onto a totally ordered group $\mathrm{G}$ and a symbol $\infty$, such that for all $\mathrm{x}$ and $\mathrm{y}$ in $\mathrm{K}$ :

1. $v(\mathrm{xy})=v(\mathrm{x})+v(\mathrm{y})$.

2. $v(\mathrm{x}+\mathrm{y}) \geq \min \{v(\mathrm{x}), v(\mathrm{y})\}$.

3. $v(1)=0$ and $v(0)=\infty$.

The ring $\mathrm{A}=\mathrm{A}_{v}=\{\mathrm{x} \in \mathrm{K} \mid v(\mathrm{x}) \geq 0\}$, together with the ideal $\mathrm{P}=\mathrm{P}_{v}=\{\mathrm{x} \in \mathrm{K} \mid v(\mathrm{x})>0\}$, denoted $(\mathrm{A}, \mathrm{P})$, is called a valuation pair (of $\left.\mathrm{K}\right)$. A is called a valuation ring (of $K$ ). $G$ is called the value group of $A$.

In the presence of the Marot property, valuation rings share some properties of valuation domains:

PROPOSITION $4.1[\mathrm{H}]$, [PS] Let A be a Marot ring. Assume that $A \neq Q(A)$.

Then the following conditions are equivalent:

1. A is a valuation ring. 
2. For each regular element $x \in Q(A)$, either $x \in A$ or $x^{-1} \in A$.

3. A has only one regular maximal ideal and each of its finitely generated regular ideals is principal.

Let $\mathrm{R}$ be a ring with total ring of quotients $\mathrm{Q}(\mathrm{R})$. The ideal $C(R)=\{x \in R \mid x Q(R) \subset R\}$ is called the core of $R$. Note that $C(R)$ can be obtained as the intersection of all regular ideals of $R$, or as the intersection of all regular principal ideals of $\mathrm{R}$. A valuation ring $(\mathrm{R}, \mathrm{P})$ is said to be discrete if each primary ideal $\mathrm{Q}$ of $\mathrm{R}$ such that $\mathrm{C}(\mathrm{R}) \subseteq \mathrm{Q} \subseteq \mathrm{P}$, is a power of its radical. A valuation ring $(R, P)$ has rank $\mathbf{n}$ if the rank of the value group $G$ is $n$.

A few other pertinent results concerning valuation rings will appear as we discuss generalizations of Prufer domains and Krull domains. For further results regarding valuation theory in rings with zero divisors, see, for example, [D], [H], [G5], and [PS].

Next, we briefly examine a few possible generalization of the property " $\mathrm{R}$ is a Prufer Domain" to rings with zero divisors. There are at least fourteen different characterizations of Prufer domains (see [G] and [FHP]) which may be generalized to rings with zero divisors; some of them may be generalized in several ways. There is an extensive literature exploring all generalizations available to date. We will bring up here three of the most popular generalizations. These happen to coincide with the three types of approaches mentioned in the introduction (to clarify this interpretation consider "all entities" to be "all finitely generated ideals", and see Proposition 3.11). It is instructive to see the three approaches "in action", and see how some of the zero divisor controlling conditions described in the previous sections bridge between the three generalizations. In all the three cases, we will generalize the following characterization of Prufer domains:

A domain $\mathrm{D}$ is a Prufer domain if every nonzero finitely generated ideal of $\mathrm{D}$ is invertible.

Generalizing Prufer domains using approach (a): A ring $\mathrm{R}$ is called an arithmetical ring if every finitely generated ideal of $\mathrm{R}$ is locally principal.

This kind of generalization satisfies some, but not all, of the equivalent conditions defining a Prufer domain.

PROPOSITION 4.2 [G5], [J] Let $R$ be a ring. The following conditions are equivalent:

1. $R$ is an arithmetical ring. 
2. The ideals of $R_{m}$ are totally ordered by inclusion for each maximal ideal $\mathrm{m}$. 3. The ideals of $R$ form a distributive lattice, that is, for all ideals $I, J$ and $L$ of $R$, we have $I+J \cap L=(I+J) \cap(I+L)$.

4. For all ideals $I$ and $J$, and any finitely generated ideal $L$ of $R$, we have $((I+J): L)=(I: L)+(J: L)$.

Other interesting Prufer-like properties of arithmetical rings may be found in $[\mathrm{J}]$.

Generalizing Prufer domains using approach (b): A ring is called a Prufer ring if every finitely generated regular ideal is invertible.

This definition is due to Griffin [G5], and he prefers this generalization of a Prufer domain to other generalizations, as it seems to be the one whose relation with its total ring of quotients is similar to that of a Prufer domain to its field of quotients. In [G5], Griffin exhibits 15 equivalent conditions to the property of being a Prufer ring, among them the conditions in Proposition 4.2 restricted (at least partially) to regular ideals. I find this generalization of a Prufer domain to be somewhat unsatisfactory as the similarity with a Prufer domain breaks down on an important point. A valuation ring need not be a Prufer ring $[\mathrm{BL}],[\mathrm{H}]$.

A Prufer valuation pair is closer to what one would expect a valuation pair to be, namely:

PROPOSITION $4.3[\mathrm{H}]$ Let $R$ be a ring, and let $P$ be a prime ideal of $R$. The following conditions are equivalent:

1. $(R, P)$ is a Prufer valuation pair.

2. $R$ is a Prufer ring and $P$ is the unique regular maximal ideal of $R$.

3. $R$ is a valuation ring and $P$ is the unique regular maximal ideal of $R$.

If we add the Marot condition to the definition of a Prufer ring, we eliminate most difficulties.

THEOREM 4.4 [G5], [H] Let $R$ be a Marot ring. Then $R$ is a valuation ring iff $R$ is a Prufer valuation ring.

Griffin [G5] showed that an arithmetical ring can be obtained from a Prufer ring $\mathrm{R}$ by imposing some zero divisor restricting conditions on $\mathrm{Q}(\mathrm{R})$. 
PROPOSITION 4.5 [G5] A ring $R$ is arithmetical iff $R$ is a Prufer ring and $Q(R)$ satisfies that ideals of $Q(R)_{P}$ are totally ordered by inclusion for all maximal prime ideals of zero $P$ in $Q(R)$.

Additional information about Prufer rings can be found in, for example, [H], [G5], [BL].

Generalizing Prufer domains using approach (c): $\mathrm{R}$ is a semihereditary ring, that is, every finitely generated ideal of $\mathrm{R}$ is projective.

Given that this condition implies, in particular, that principal ideals are projective, the zero divisor controlling condition imposed with this generalization is the PP condition. We will actually see that the zero divisor controlling condition of this generalization can be viewed in a different way.

THEOREM 4.6 [G1], [G5], [M1] Let $R$ be a ring. The following conditions are equivalent:

1. $R$ is semihereditary.

2. $R$ is coherent and w.gl.dim $R \leq 1$.

3. $Q(R)$ is Von Neumann regular and $R_{m}$ is a valuation domain for every maximal ideal $m$ of $R$.

4. $R$ is a Prufer ring and $Q(R)$ is Von Neumann regular.

A semihereditary ring $\mathrm{R}$ shares the following property with a Prufer domain: $\mathrm{R}_{\mathrm{P}}$ is a valuation domain for all prime ideals $\mathrm{P}$ of $\mathrm{R}$.

Marot's investigation [M1] into the zero divisor controlling condition " $Q(R)$ is Von Neumann regular" not only yielded the rich characterization of semihereditary rings of Theorem 4.6 (3), but also added an equally useful and interesting characterization of hereditary rings.

Theorem 4.7 [G1], [M1], [V] Let $R$ be a ring. Then $R$ is hereditary iff $Q(R)$ is hereditary and any ideal of $R$ that is not contained in any minimal prime ideal of $R$ is projective.

Additional results about semihereditary rings and related homological conditions can be found in [G1] and [V]. 
We next examine some aspects of the generalization of the concept of being a Krull domain to rings with zero divisors. Let $R$ be a ring and let $Q(R)$ be the total ring of quotients of $R$. Assume $R \neq Q(R)$. (This is a technical condition making the statements of some theorems cleaner and the statements of other theorems messier. Some authors prefer to make this distinction, other prefer not to.) $\mathrm{R}$ is called a Krull ring if there exists a family $\left\{\left(\mathrm{V}_{\alpha}, \mathrm{P}_{\alpha}\right) \mid \alpha \in \mathrm{I}\right\}$ of discrete rank one valuation pairs of $Q(R)$ with associated valuations $\left\{v_{\alpha} \mid \alpha \in I\right\}$ such that: 1. $\mathrm{R}=\cap\left\{\mathrm{V}_{\alpha} \mid \alpha \in \mathrm{I}\right\}$.

2. $v_{\alpha}(a)=0$ for almost all $\alpha$ for each regular element a $\in Q(R)$, and each $P_{\alpha}$ is a regular ideal of $\mathrm{V}_{\alpha}$.

There are three definitions of a Krull ring with zero divisors in the literature. Our definition is by Kennedy [K4], and was also adopted by Kang [K], [K1], [K2]. Another definition is by Huckaba [H]. Huckaba's definition is identical with a Marot Krull ring in the Kennedy sense. The third definition is by Portelli and Spangler [PS]. Portelli and Spangler's definition can be shown to be equivalent to Huckaba's definition.

Perhaps here is the place to say that adding the Marot condition to the definition of a Krull ring brings this generalization closer to sharing many of a Krull domain properties. For example, even the definition itself becomes smoother as the notion of a rank 1 discrete valuation ring is more manageable: A Marot valuation ring $(\mathrm{V}, \mathrm{P})$ with associated valuation $v$ and value group $\mathrm{G}$ is a discrete rank one valuation ring (respectively, $v$ is a discrete rank one valuation) if $\mathrm{G}$ is isomorphic to the group of integers. In this case, $\mathrm{P}$ is the unique regular prime ideal of $\mathrm{V}$ and there exists a regular element $\mathrm{x}$ of $\mathrm{P}$ such that $\mathrm{P}=(\mathrm{x})$. Marot Krull rings share many properties of Krull domains. $[\mathrm{H}]$ provides a particularly clear and detailed exposition of these results. [K], [AM], and [AM1] take up the notion of a UFD and extend it (in a number of ways) to rings with zero divisors. It is the Marot property that allows for the conclusion of Corollary 3.13. As a consequence, only under the additional assumption that a ring is both Marot and Krull do the authors get a relation between factoriality properties and Krull ring behavior reminiscent of the domain case. A good survey article on the extension of the UFD notion to rings with zero divisors is [A].

It is possible to define Krull rings without resorting to valuation rings.

Recall that a ring $R$ is completely integrally closed if, for $0 \neq a$ and $u$ in $\mathrm{Q}(\mathrm{R}), \mathrm{au}^{\mathrm{n}} \in \mathrm{R}$ for all $\mathrm{n}$ implies $\mathrm{u} \in \mathrm{R}$.

Let $\mathrm{I}$ be a nonzero fractional ideal of R. $\mathrm{I}_{\mathrm{t}}$ is defined to be $\Sigma\left(\mathrm{I}_{0}^{-1}\right)^{-1}$, 
where $\mathrm{I}_{0}$ runs over the nonzero finitely generated $\mathrm{R}$ submodules contained in $\mathrm{I}$. We say that $\mathrm{I}$ is $\mathbf{t}$ invertible if $\left(\mathrm{II}^{-1}\right)_{\mathrm{t}}=\mathrm{R}$.

THEOREM 4.8 [H], [K2], [M4] The following conditions are equivalent for a ring $R$ :

1. $R$ is a Krull ring.

2. [M4] $R$ is completely integrally closed and satisfies the ascending chain condition on divisorial ideals.

3. [K2] Every regular ideal of $R$ is $t$-invertible.

4. [K2] Every regular prime ideal of $R$ is $t$ invertible.

5. [K2] Every regular prime ideal of $R$ contains a tinvertible regular prime ideal.

All references mentioned above contain additional information on Krull rings.

A related topic is the investigation into the behavior of the integral closure of a ring with zero divisors. Tom Lucas and others have done fundamental work in this direction. Interested readers are referred to [L] for a good survey and additional references on this topic.

\subsection{Finite Conductor Rings and G-GCD Rings}

The next application of the zero divisor controlling conditions described in the previous sections involves the use of the PP condition in a recent generalization by Glaz [G3], [G4] of finite conductor properties to rings with zero divisors.

Let $R$ be a ring. $R$ is a finite conductor ring if (a) $\cap(b)$ and (0:c) are finitely generated ideals of $\mathrm{R}$ for all elements $\mathrm{a}, \mathrm{b}$, and $\mathrm{c}$ of $\mathrm{R}$. $\mathrm{R}$ is a quasicoherent ring if $\left(a_{1}\right) \cap \ldots \cap\left(a_{n}\right)$ and $(0: c)$ are finitely generated for all elements $\mathrm{a}_{1}, \ldots, \mathrm{a}_{\mathrm{n}}$ and $\mathrm{c}$ of $\mathrm{R}$.

This definition [G3] extends the notion of finite conductor and quasicoherence of domains by adding the zero divisor controlling condition: " $(0: c)$ is a finitely generated ideal for all c". Though this is a much weaker condition than the PP condition, it does affect the domain-like behavior of some rings. For example:

PROPOSITION 4.9 [G3] Let $R$ be a ring with weak global dimension one. The following conditions are equivalent:

1. $R$ is a semihereditary ring. 
2. $R$ is a coherent ring.

3. (0:c) is a finitely generated ideal of $R$ for every element $c$ of $R$.

With these definitions finite conductor and quasi-coherent rings accept several equivalent domain-like characterizations [G3], [G4].

A particular case of a finite conductor ring is the recently defined GGCD ring. A G-GCD domain is defined by the condition that intersections of two invertible ideals is an invertible ideal [AA]. Glaz [G3], [G4] generalized this condition to rings with zero divisors as follows:

A ring $\mathrm{R}$ is called a G-GCD ring if the following two conditions hold: 1. $\mathrm{R}$ is a PP ring.

2. The intersection of any two finitely generated flat ideals of $R$ is a finitely generated flat ideal of $\mathrm{R}$.

At first glance, it seems that one may replace condition 2 by other, similar conditions and obtain different generalizations of the G-GCD domain notion. But in fact the PP condition is powerful enough to make all these generalizations coincide:

THEOREM 4.10 [G3], [G4] Let $R$ be a ring. The following conditions are equivalent:

1. $R$ is a $G-G C D$ ring.

2. $R$ is a PP ring and the intersection of any two principal (fractional) ideals of $R$ is a finitely generated flat (fractional) ideal of $R$.

3. $R$ is a PP ring and the intersection of any two finitely generated projective ideals of $R$ is a finitely generated projective ideal of $R$.

4. $R$ is a PP ring and the intersection of two invertible ideals of $R$ is an invertible ideal of $R$.

G-GCD rings are reduced rings which are locally GCD domains, they are integrally closed in their total ring of quotients, and they possess compact $\operatorname{Min}(\mathrm{R})$ and Von Neumann regular total ring of quotients. Coherent regular rings are G-GCD rings, but not all G-GCD rings are coherent [G3]. On the other hand, it was through this definition that coherent-like and regularity-like properties of polynomial rings over coherent rings were discovered. Namely:

THEOREM 4.11 [G3], [G4] 
1. Let $R$ be an integrally closed coherent domain. Then the polynomial ring $R[x]$ is a quasi-coherent domain.

2. Let $R$ be a coherent regular ring. Then the polynomial ring $R[x]$ is a $G-G C D$ ring.

For additional properties of finite conductor, quasi-coherent, and G-GCD rings see [G3], [G4]. A different definition of a G-GCD ring, and a study of this class of G-GCD rings, appear in Ali and Smith [AS].

\subsection{Going Down and Related Rings}

Another application of the zero divisor controlling conditions described in the previous sections is the use of the condition $\mathrm{Nil}(\mathrm{R})=\mathrm{Z}(\mathrm{R})$ for the extension of several related domain conditions to rings with zero divisors.

A ring extension $\mathrm{R} \subseteq \mathrm{T}$ satisfies Going Down (GD) if given prime ideals $\mathrm{P} \subset \mathrm{P}_{1}$ in $\mathrm{R}$, and $\mathrm{Q}_{1}$ in $\mathrm{T}$ satisfying $\mathrm{Q}_{1} \cap \mathrm{R}=\mathrm{P}_{1}$, there is a prime ideal $\mathrm{Q}$ in $\mathrm{T}$ such that $\mathrm{Q} \subset \mathrm{Q}_{1}$ and $\mathrm{Q} \cap \mathrm{R}=\mathrm{P}$. A domain $\mathrm{R}$ is called a Going Down domain in case the extension $\mathrm{R} \subseteq \mathrm{T}$ satisfies GD for each overring $\mathrm{T}$ of $\mathrm{R}$. Dobbs [D1] extended the Going Down notion to rings with zero divisors as follows: A ring $\mathrm{R}$ is a Going Down ring if $\mathrm{R} / \mathrm{P}$ is a Going Down domain for every prime ideal $\mathrm{P}$ of $\mathrm{R}$.

Under the zero divisor controlling condition $\mathrm{Nil}(\mathrm{R})=\mathrm{Z}(\mathrm{R})$, this notion becomes a natural generalization of the Going Down property for domains.

PROPOSITION 4.12 [D1] Let $R$ be a ring in which 0 is a primary ideal. Then $R$ is a Going Down ring iff $R \subseteq T$ satisfies GD for each overring $T$ of $R$.

Examples are provided in [D1] that show that the assumption $\operatorname{Nil}(\mathrm{R})=\mathrm{Z}(\mathrm{R})$ is necessary.

Two related notions in which the condition $\operatorname{Nil}(\mathrm{R})=\mathrm{Z}(\mathrm{R})$ played a role are the notions of divided and locally divided rings introduced in [B] and [BD]. $\mathrm{A}$ ring $\mathrm{R}$ is a divided ring (respectively, a locally divided ring) if each prime ideal is comparable under inclusion with each ideal of $R$ (respectively, if $R_{P}$ is a divided ring for every prime ideal $\mathrm{P}$ of $\mathrm{R}$ ). Divided rings are Going Down rings, though the converse is false even for domains [D1]. David Anderson, Badawi and Dobbs [BAD], [ABD] extended another domain notion to rings with zero divisors, namely the PVD notion. PVDs were first defined by Hedstrom and Houston in $[\mathrm{HH}]$ as those domains for which every prime ideal is strongly prime. 
In a domain $\mathrm{D}$ a prime ideal $\mathrm{P}$ is strongly prime if $\mathrm{xy} \in \mathrm{P}$, for $\mathrm{x}$ and $\mathrm{y}$ in the field of quotients of $\mathrm{D}$, implies $\mathrm{x} \in \mathrm{P}$ or $\mathrm{y} \in \mathrm{P}$. This notion was extended to rings with zero divisors as follows: Let $\mathrm{R}$ be a ring. A prime ideal $\mathrm{P}$ of $\mathrm{R}$ is said to be strongly prime if $\mathrm{aP}$ and (b) are comparable for all $\mathrm{a}, \mathrm{b} \in \mathrm{R}$. A ring is called a pseudo valuation ring (PVR) if each prime ideal is strongly prime. It is interesting to note that if $(R, m)$ is a local PVR, then $Z(R)$ can be Nil(R), $m$, or any prime ideal properly in between $[\mathrm{ABD}]$. A detailed analysis of these rings can be found in $[\mathrm{B}],[\mathrm{BD}]$, [BAD], and [ABD].

\section{REFERENCES}

[A] D. D. Anderson, Extension Of Unique Factorization: A Survey, Marcel Dekker Lecture Notes in Pure Appl. Math. 205 (1999), 31 - 53

[AA] D. D. Anderson and D. F. Anderson, Generalized GCD Domains, Comm. Math. Univ. St. Pauli XXVIII (1979), 215 - 221

[ABD] D. F. Anderson, A. Badawi and D. E. Dobbs, Pseudo Valuation Rings, II, Bollettino U. M. I. (8) 3-B (2000), 535 - 545 [AM] D. D. Anderson and R. Markanda, Unique Factorization Rings With Zero Divisors, Houston J. Math. 11 (1985), 15 - 30 [AM1] D. D. Anderson and R. Markanda, Unique Factorization Rings With Zero Divisors: Corrigendum, Houston J. Math. 11 (1985), 423 426

[AS] M. M. Ali and D. J. Smith, Generalized GCD Rings, Beiträge zur Algebra und Geometrie 42 (2001), 219 - 233

[B] A. Badawi, On Divided Commutative Rings, Comm. Algebra 27 (1999), $1465-1474$

[BAD] A. Badawi, D. F. Anderson and D. E. Dobbs, Pseudo Valuation Rings, Marcel Dekker Lecture Notes in Pure Appl. Math. 185 (1997), 57 $-67$

[BD] A. Badawi and D. E. Dobbs, On Locally Divided Rings And Going Down Rings, Comm. Alg., to appear

[BL] M. B. Boisen and M. D. Larsen, Prufer And Valuation Rings With Zero Divisors, Pacific J. Math. 40 (1972), 7 - 12

[D] E. D. Davis, Overrings Of Commutative Rings II, Transactions Amer. Math. Soc. 110 (1964), 196 -212

[D1] D. E. Dobbs, Going Down Rings With Zero Divisors, Houston J. Math. 23 (1997), 1 - 11

[E] S. Endo, On Semihereditary Rings, J. Math. Soc. Japan 13 (1961), 109 -

119

[E1] E. G. Evans, Zero Divisors In Noetherian Like Rings, Trans.

American Math. Soc. 155 (1971), 505 - 512 
[FHP] M. Fontana, J. A. Huckaba and I. J. Papick, Prufer Domains, Marcel Dekker (1997)

[G] R. Gilmer, Multiplicative Ideal Theory, Queen's Papers on Pure Appl. Math. 12 (1968)

[GH] R. Gilmer and J. A. Huckaba, $\Delta$ Rings, J. Algebra 28 (1974), 414 - 432

[GH1] R. Gilmer and W. Heinzer, The Laskerian Property, Power Series

Rings And Noetherian Spectra, Proc. American Math. Soc. 79

(1980), 13 - 16

[G1] S. Glaz, Commutative Coherent Rings, Springer Verlag Lecture Notes in Math. \# 1371, (1989)

[G2] S. Glaz, Commutative Coherent Rings: Historical Perspective And Current Developments, Nieuw Arch. Wiskunde 10 (1992), 37 - 56

[G3] S. Glaz, Finite Conductor Rings, Proc. American Math. Soc. 129 (2001), 2833 - 2843

[G4] S. Glaz, Finite Conductor Rings With Zero Divisors, Kluwer

Academic Publisher Math and Appl. \# 520 (2000), 251 - 271

[G5] M. Griffin, Prufer Rings With Zero Divisors, J. Reine Angew.

Math. 239/240 (1970), 55 - 67

[H] J. A. Huckaba, Commutative Rings With Zero Divisors, Marcel

Dekker, Inc. (1988)

[H1] J. A. Huckaba, On Valuation Rings That Contain Zero Divisors, Proc.

Amer. Math. Soc. 40 (1973), 9 - 15

[HH] J. R. Hedstrom and E. G. Houston, Pseudo-Valuation Domains, Pacific J. Math. 75 (1978), 137 - 147

[HO] W. Heinzer and J. Ohm, On The Noetherian Like Rings Of E. G.

Evans, Proc. American Math. Soc. 34 (1972), 73 - 74

[J] C. U. Jensen, Arithmetical Rings, Acta Math. Acad, Sci.

Hungaricae 17 (1966), 115 - 123

[K] B. G. Kang, A Characterization Of Krull Rings With Zero

Divisors, J. Pure Appl. Algebra 72 (1991), 33 - 38

[K1] B. G. Kang, Integral Closure Of Rings With Zero Divisors, J.

Algebra 162 (1993), 317 - 323

[K2] B. G. Kang, Characterizations Of Krull Rings With Zero

Divisors, J. Pure Appl. Algebra 146 (2000), 283 - 290

[K3] I. Kaplansky, Commutative Rings, Allyn and Bacon, Inc. (1970)

[K4] R. E. Kennedy, Krull Rings, Pacific J. Math. 89 (1980), 131 - 136

[L] T. G. Lucas, Integrality Properties In Rings With Zero Divisors,

Marcel Dekker Lecture Notes Pure Appl. Math. 220 (2001), 265 - 280

[M] M. E. Manis, Extension Of Valuation Theory, Bull. Amer. Math. Soc. 73 (1967), 735 - 736

[M1] J. Marot, Ph.D Thesis: Sur Les Anneaux Universeillement

Japonais, Univ. Paris-Sud (1977) 
[M2] E. Matlis, The Minimal Prime Spectrum Of A Reduced Ring,

Illinois J. Math. 27 (1983), 353 - 391

[M3] R. Matsuda, On Marot Rings, Proc. Japan Acad. 60 (1984), 134 - 137

[M4] R. Matsuda, On Kennedy's Problem, Comm. Math. Univ. Sancti Pauli 31 (1981), 13 - 24

[O] J. P. Olivier, Anneaux Absolument Plats Universels Et Épimorphismes D'Anneaux, C. R. Acad. Sci. Paris Ser. A 266 (1968), 317 - 318

[O1] J. P. Olivier, Anneaux Absolument Plats Universels Et Épimorphismes Á Buts Reduits, Sém. Samuel, Paris (1967 - 1968), 6.1 $-6.12$

[PS] D. Portelli and W. Spangler, Krull Rings With Zero Divisors, Comm. Algebra 11 (1983), 1817 - 1851

[Q1] Y. Quentel, Sur La Compacité Du Spectre Minimal D’un Anneau, Bull. Soc. Math. France 99 (1971), 265 - 272

[Q2] Y. Quentel, Erratum, Sur La Compacité Du Spectre Minimal D'un Anneau, Bull. Soc. Math. France 100 (1972), 461

[V] W. V. Vasconcelos, The Rings Of Dimension Two, Marcel Dekker Lecture Notes in Pure Appl. Math \# 22 (1976) 